\title{
Aspecto del Nervio Óptico en la Detección del Glaucoma
}

\author{
RUTY TERRY \\ Deparamenso de Ofubmología. Centro de Reconocimiento Médico PNP-DISAN. \\ Hospiral Central de la Policia Nacional del Perí.
}

\begin{abstract}
RPAUMIEN
Entre diciembre de 1995 y mayo de 1996 se evaluó 725 pacientes en el Servicio de Oftalmología del Hospital

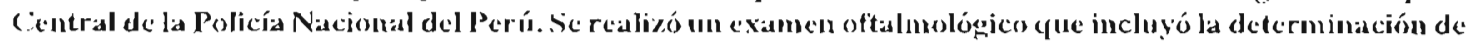
la presión intraocular, la evaluación del aspecto del nervio óptices y del campo visual. Se cencontró una casuística de $1,96 \%$ de glaticonla recién diagnosticado, $12,27 \%$ de hipertensos nculares y $6,71 \%$ de pacientes con relación pseudoglaucomatosa del disco. La húscfueda de glancoma en bas campañas de salud ocular, que se hasa cxclusivamente c'n valores clevados de la presión intraocular, puede mejorar significativantente su sensibilidad y especifícidad si se evalía además el aspecto del nervio óptico.
\end{abstract}

Palabras claves: Glaucoma; P'resion Intraocular; Nervio Óptico.

OPIIC NISRVE APPEARANCE FOR GLAUCONLA DETLCIION SUMILAKY

Between Decenber 1995 and May 1996, 725 paticnts underwent an evaluation at the Department of Ophthalnology, Hospital Central de la Policía del Perí. 'The ophthalmologic examination included measurements of intraocular presiure (IOP), visilal fields, and optic nerve appearance. Wic found recently diagngosed plaucoma incidence of 1,96\% a high IOI' in 12,27\% patients, and 6,71\% with psendoglaticonatous disc ratio. Sercening programs for massive detection of glaucoma hased on I()P as a single parameter can improve their sensitivity and specificity by adding the optic nerve appearance assessment.

Key words: Glaucoma; Intraocular Pressure; Optic Nerve.

\section{INTRODUCCIÓN}

El glaucoma es una de las causas nás frecuentes de déficit visual en adultos. siendo el curso clínico progresivo e inicialmente asintomático. En cesta líl tima característica radica su

\section{Correspondencia:}

Dra. Run: Term Ponte.

Deparantento de Ofralmologia.'

Hospital Cenural P:VP.

Ar: Brasil Cumedra 26.

Lima N . Perin. importancia, pues muchos pacicntes acuden por atcución médica en estadios avanzados de la enfermedad. cuando la pérdida visual ha alcankado niveles de irrecuperahilidad (1.2).

El glaucoma constituye la segunda causa de ceguera en los Estados Unidos ( ${ }^{3}$ ) y es molivo irecuente de consula olialmológica por ser má en fernuedad crónica que ocisiona deterioro en la calidad de vida del paciente, alectando su productividad por los años-ciceno perdidos y por el allocosto del tratamiento $\left(^{4}\right)$.

Clásicantente cl glaucona sc relacioma con niveles ckvados de la presion intracular (PIO). por lo que este lador 
ha sido enfatizado aisladamente en al gunas investigaciones nacjonales ( $\left.{ }^{5-7}\right)$. Sin emhargo, en la actualidad se considera que la detección de gliucoma en campañas de salud pública basada únicamente en la determinación de una presión intraocular elevada mo) sería suficientemente contiable $\left(^{(5.5)}\right.$.

El objetivo de esta investigacjón fue descubrir en una poblacion de pacientes del Hospital Central de la Policía Niacional del Penú, la disuribucion de los niveles de la presión intraocular y su relación con el aspecto del nervio óptico, y evaluar su importancia como indicadores en la detección de glaucoma cn campañas de salud ocular.

\section{MATERIAL, Y MÉTODOS}

Durante el períndo comprendido entre dicientbre de 1995 y mayo de 1996 se evaluo 725 pacientes de ambos sexos entre 7 y 99 años de edad que residran en dilerentes distritos de Lima y de toda condicionsocioecorónica, quc acudían al Consulkorio Externo de Oltadmología del Hospital Central de la Policía Nacional del Perú.

Los pacientes en su mayoría cran amétropes que acudían para un exancen de refración. No participaron pacientes con patología ocular intlanatoria.

\section{Procedimiento}

Se siguio un protocolo que incluyo fỉliación con inclusion de edad. sexo, raza, procedencia. antecedentes de diagnóstico y tratamicnto de glaucoma e historia familiar. En el exancon of talmológico se evaluó: agudcra visual. examen del segmento anterior, gonioscopia, Condo de ojo por oflalmoscopía directa. campos visuales y retinografía bidimensional a colores del nervio óptico.

Se emples un Neumotonómetro Topcon Mod. CT-20, previamentc calibrado, para la determinación de la presión intraocular, entre las 08:00 h y las 13:00 h. Con el paciente sentado y sin colocación de anestesia tópica, se realizó la tonometría, en primer lugar en el ojo derecho (OD) y luego en el ojo izquierdo (OI). Se obluvo 3 determinaciones por cada ojo y se calculs un primer promedio de la PIO. Inmediatamente se realizó el londo de ojo sin conocer el resultado tonométrico. Si el aspecto de la papila era sospechoso de glaucoma se tomaba und retinografía bidimensional del nervio optico haciendo uso de una cámara retinal Opcon RC-50f y película Kodak Ektachrome $100^{\mathrm{*}}$. Posteriomente, las fotografías eran examinadas en husca de características de glaucoma

A los pacientes que presentaban el primer promedio de PIO mayor or igual a $22 \mathrm{~mm} H \mathrm{Hg}$ y/o papila sospechosa de glaucoma. sc les determinaba nuevamente la PIO después de 7 dias obteniendose un segundo promedio de la PIO de aunbos ojos. La semisuma de ambos valores proporcionó un valor final para la PIO. Si estal citra era mayor o igual a $22 \mathrm{mmHg}$, era considerada como Hipertensión Ocular (HTO). A todos los pacicntes que presentaron HT(3 y/o papila sospechosa se les réaliró un campo visual con un Perímetro manual MK-70ST. Posteriomiente se realizaba el examen del segmento anterior con Limmpara de Hendidura y Gonioscopía (Fig. No 1 ).

\section{Criterios Diagnósticos}

Se determino el diagnóstico de glaucoma si el paciente presentaba por lo menos 2 de las 3 características siguicnles:

1.- La apariencia del disco: relación copaldisco (RCD) > 0,3 cn el eje vertical o asimetría de $(0,2$ en el ojo comprometido, adel gazamiento del borde o hemorragia de la papila (disco con sospecha de glaucoma).

2.- Presión intraocular mayor o jgual a $22 \mathrm{~mm} H \mathrm{Hg}$ (Hipertensión Ocular).

3.- Delecto del campo visual compatible con glaucoma: defecto arcuato. escakón nasal, escotoma paracentral. depresión general cn ausencia de otras causas que expliquen estas alteraciones (campo visual anormal).

El glaucoma de tensión normal se definnió por la presencia de los criterios 1 y $3(1+., 9)$.

\section{R ISULTADOS}

De los 725 pacientes evaluados, se excluyo 114 individuos que presentaron los siguientes diagnosticos, entre otros: 26 cataratas. 10 leucomas, 6 pterigion invasivos, 2 desprendimientos de retina. 4 hialosis asteroideas que no tenran antecedentes de glaucoma, 1 obstrucción de la vena central de la retina, otros diagnosticos: 6. No completaron el protocolo: 6 pacientes. 


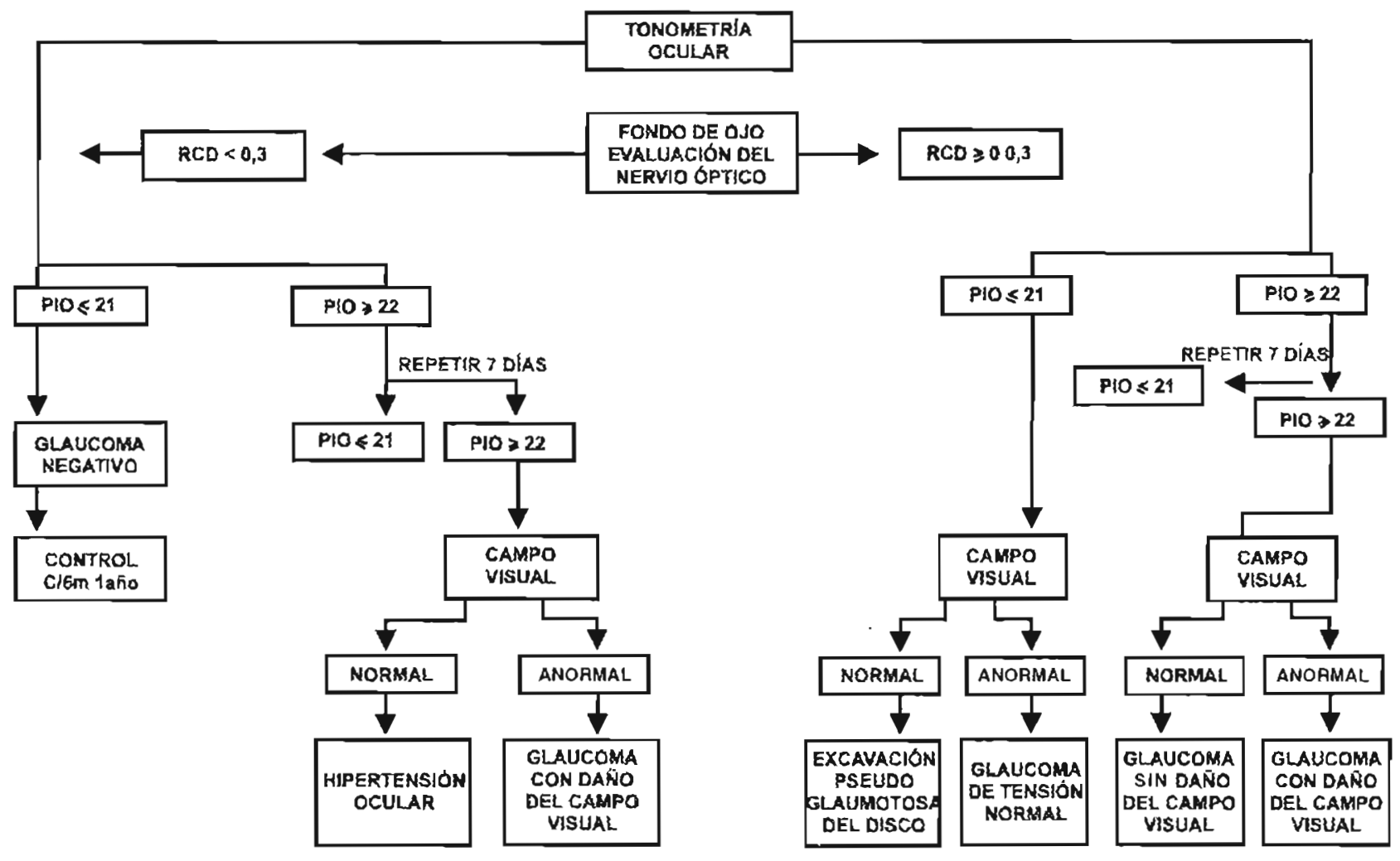

Fig. No 1.- Metodología de investigación: rastreo público de glaucoma.

Se evaluo rinalmente 1222 ojos correspondientes a 611 pacientes. Pertoncieron al sexo masculino $175(28.64 \%)$ y $4.36(71,35 \%)$ al temenino.

La distribucion etárea de la población fue la siguiente: contrc $7-14$ años. 9 ( $1.47 \%$ ): 15-30 años, 79 (12.93\%): $31-45$ aปtus. 133 (21.77\%): 46-60) años. 208 (34\%): y mayores de 60) años, 182 (29.79\%) (Tabla $N^{\circ} 1$ ). El 99, $1 \%$ de los pacientes correspondieron a raza mestiza.

El $98.7 \%$ de los pacientes procedían de diferentes distritos de Lima. y $1.3 \%$ (8 pacientes) procedían de fuera de Lima.

\section{Distribución de las Presiones Intraoculares}

\section{En el ojo derecho:}

La mayor frouencia se encuentra en los grupos de $16-18$ $m m \mathrm{Hg}\left(30.93 \%\right.$ y de $19.21 \mathrm{mmHg}(29.3 \%)\left(\right.$ Figura $\left.N^{n} 2\right)$.

\section{En el ojo izquierdo:}

El mayor porcentaje se encuentsa en los grupos de 16-18 $\mathrm{mmHg}(30.93 \%)$ y de $19-21 \mathrm{mmHg}(27.82 \%)$ (Figura $\mathrm{N}^{0} 3$ ).

No existen difercncias signilicativas entre ambos ojos

Relación entre la Presión Intraocular y el Sexo Sexo Masculino:

La PIO or lise de $17,366 \pm 3,396 \mathrm{mmHg}$ y la $\mathrm{PIO}_{\text {ol }}$ fuc de $17.589 \pm 3,487 \mathrm{mmHg}$.

\section{Sexo Fentenino:}

La $\mathrm{PIO}_{(3)}$ tuc de $17.447 \pm 3.609 \mathrm{mmHg}$ y' la $\mathrm{PIO}_{(01}$ liue de $17.339 \pm 3,506 \mathrm{~nm} m \mathrm{Hg}$.

No existen diferencias kntro ambos sexos. 


\section{Distribución de la Relación Copa/Disco}

Sexo Masculino:

La relación copaddisco en el ojo derecho fue de $0.254 \pm$ 0.097 y en el ojo izquierdo de $0,253 \pm 0,096$ (Fig. 4 y 5).

\section{Sexo fiemenimo:}

La relación copa/disco en el ojo derecho tue de 0.239 \pm 0,112 y en el ojo izquicrdo de $0,236 \pm 0,103$.

Se ohserva que no existe diforencia significativa entro ambos sexos.

\section{Relación entre la Relación Copa/Disco y la Edad}

El análisis de corrclación y regresión para $p=0,05$ demuestra que existe una relación directa y estadísticamente significativa que secumplecon la población tolial y el sexo porseparado.

\section{Relación entre la PlO y la Relación Copa/Disco}

El análisis de correlación y regresión para $p=0.05$ demuestra que ambas variables están relacionadas directa- mente y en forma significativa cuando se evalúa a la población tolal.

\section{Casuítica de Claucoma, Hipertensión Ocular y Excavación Pseudoglaucomatosa del Disco}

Se encontró 12 paciéntes con glaucoma: 6 con compromiso de ambos ojos y 6 unilateralex. Si los clasilicamos por cl grado de hipertension ocular, se hallo 9 pacientes con PIO mayor o igual a $22 \mathrm{~mm} H \mathrm{Hg}$ y 3 casos de glaucoma a tensión nomial.

Sc obuvo una casuística de 1,96\% de pacientes con glaucoma crónico; de ellos, como se nencionó antes, la tercera parte corresponde a glatucomas con PIO menor o igual a 21 $\operatorname{minH}$

Presentaron hipertensión ocular 75 pacientes ( $12.27 \%)$. de los cuales presentiron HTO $: 23(3,766 \%)$. HTO or: 26 $(4,25 \%)$ e HTO $\mathrm{An}: 26(4,25 \%)$.

Se halló 41 pacientes con excavación pseudoglaucomalosa del disco $(6,71 \%)$, de los cuales fueron solo del ojo derecho: $5(0,82 \%)$, del ojo iz.quierdo: $5(0,82 \%)$, y de ambos ojos: $31(5,07 \%)$.

Tabla $\mathbf{N}^{\circ}$ 1.- Distribución de pacientes según edad y’ sexo.

\begin{tabular}{|c|c|c|c|c|c|c|}
\hline \multirow[t]{2}{*}{ Edad } & \multicolumn{2}{|c|}{ Total } & \multicolumn{2}{|c|}{ Masculino } & \multicolumn{2}{|c|}{ Fentenino } \\
\hline & $n$ & $\%$ & $n$ & $\%$ & $n$ & $\%$ \\
\hline $7-14$ & 9 & 1,47 & 2 & 1,14 & 7 & 1.61 \\
\hline $15-30$ & 79 & 12.93 & 25 & 14.29 & 54 & 12.39 \\
\hline $31-45$ & 133 & 21.77 & 18 & 10.29 & 115 & 26.38 \\
\hline $46-60)$ & 208 & 34.04 & 51 & 29,14 & 157 & 36,01 \\
\hline$>60$ & 182 & 29,79 & 79 & 45,14 & 103 & 23.62 \\
\hline Tolal & 611 & 100.00 & 175 & 100,00 & 436 & 100,00 \\
\hline
\end{tabular}




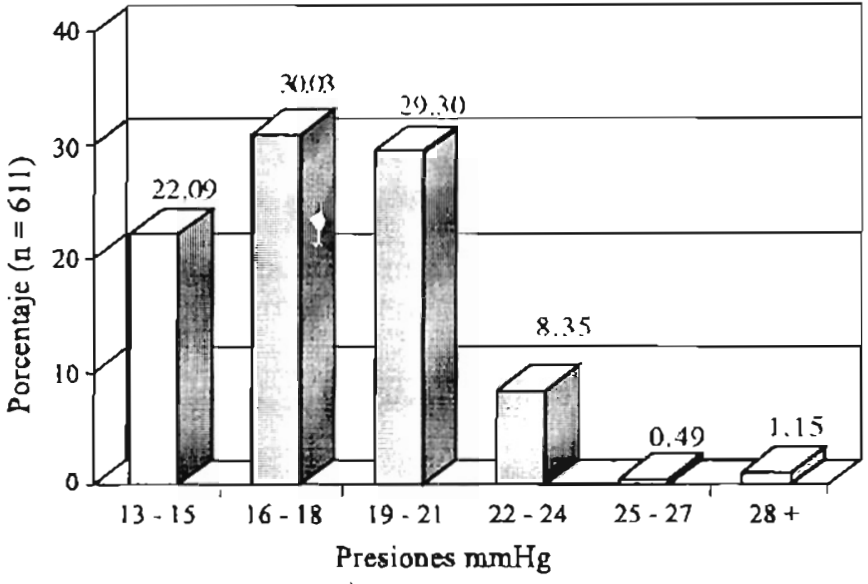

lïg. $N^{\circ}$ 2.- Distribución de PIO en ojos derechos,

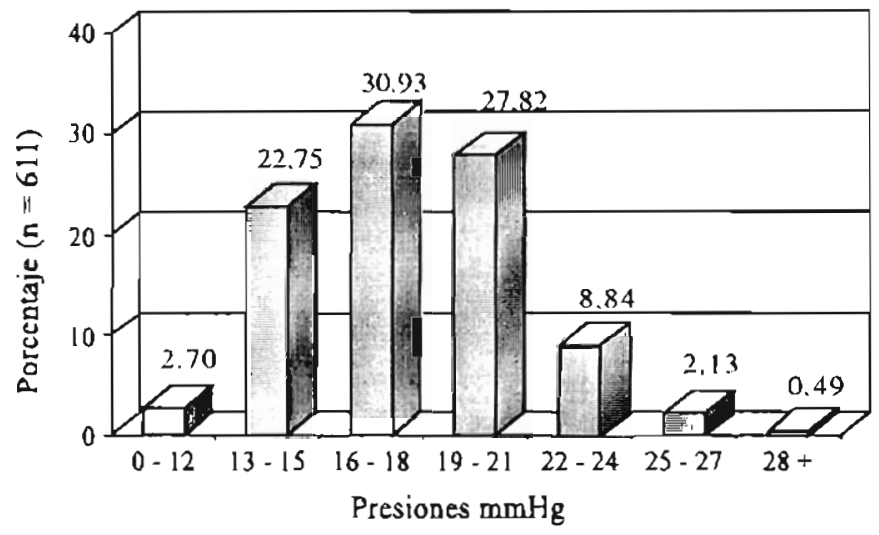

Fig. N"3.- Distribución de PIO en ojos izquierdos.

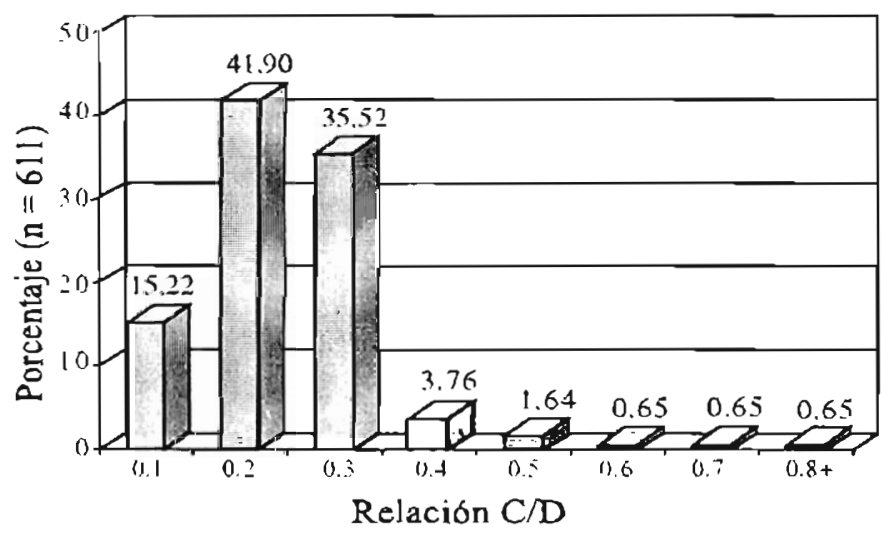

Fig. $N^{\circ}$ 4.- Distribución de la relación vertical copa/disco (C/D): ojo derecho

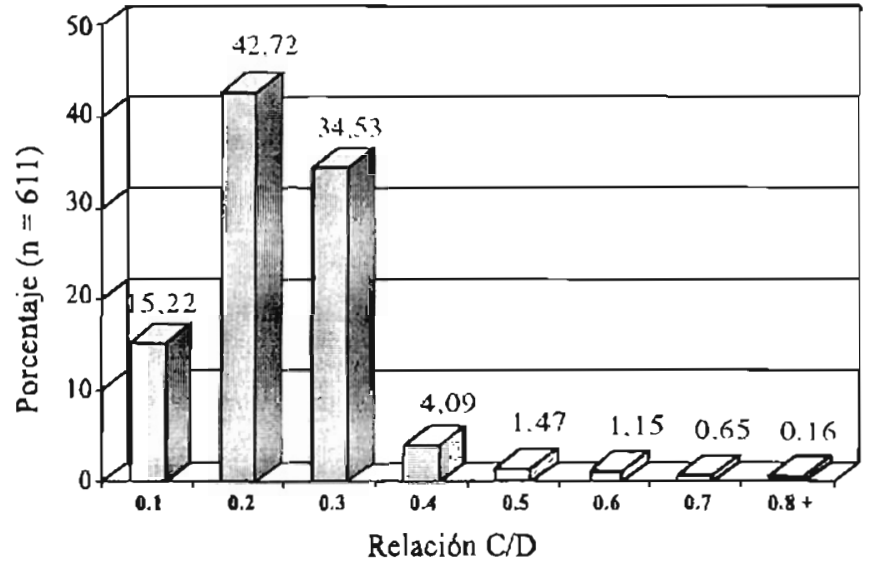

Fig. N"5.- Distrihución de la relación vertical copa/disco (CDD). ojoizcpuicudo.

\section{DISCUSIÓN}

El predominio del sexo lemenino y la menor proporción de pacientes cntre 31 y 4.5 años de edad pucke explicarse por el horario matutino establecido pasa la deleccion de los casos. que correspondio con el horario laborable. El signilicado clínico de las vatriaciones diurnas de la presión intraocular señala que la lonometría en las mananas scría la más representativa $(+.5 .10)$.

A diferencia de investigaciones anteriores que cmplearon el tonónetro de applanación de (ioldmanno el de indentación

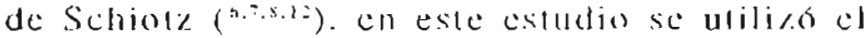
Neumolonómetro, que lienc la venlaja de ser un mélodo no invasivo. habiéndose demostrado yue los valores obtenidos son equivalentes al compararlos con los del tonóneles de aplanación de Goldmann. La distribución de los valores de la PIO es muy similar a la deotras investigandones y los promkdios de presión intrikcular (PIO) $: 17.424 \mathrm{~mm} H \mathrm{Hg}$ y: $\mathrm{PIO}_{(, 1)}: 17.5 .33$ $\mathrm{nm} \mathrm{Hg}$ ) comparahles a estudios natcioniales ("i).

No se encontró diferencia entre las presiones oculares de ambos ojos. lo que coincide cinn lo descrito por Leste (") $y^{\prime} \operatorname{Klcin}\left({ }^{15}\right)$.

Si bien existe una tendencia al aumento de la presión intracular con lat edad. no se demencstra que ésta tenga una relación significativa. a diferencia de lo descrito por Leske (").

La relacion entre la RCD y la edad fue estadísticamente signifuativa así como la relación entre la RCD y la PIO: lo que conincide con lo descrito por Shields (') y la AAO (i). 
La casuística de glaucoma fue de $1,96 \%$ (casos recién diagnosticados), similar a las reportadas por Wong $1,48 \%$ ( $)$, Oyola $1,15 \%$ () y Rosas $2,8 \%\left({ }^{3}\right)$. Klein $\left({ }^{13}\right)$ reliere $2,1 \%$ en estudios de prevalencia.

Cabe resaltar que la tercera parte de los casos de glaucoma se registró con niveles de PIO menores o iguales a $21 \mathrm{mmHg}$, estos resultados son comparables a los referidos por Leske $\left({ }^{3}\right)$, quen señala que entre la sexta park y la mitad de casos de glaucoma crónico cursan con PIO $<22 \mathrm{mmHg}$.

Sorprende la elevada frecuencia de HTO obtenida. que fue $12,27 \%$ (casos unilaterales y bilaterales): mucho mayor que la señalada por la AAO $\left({ }^{14}\right)$ de 4 a $7 \%$. Wong reporto $5,06 \%\left({ }^{6}\right)$, Oyola $1,74 \%\left({ }^{7}\right)$ y Rosas $1,81 \%\left({ }^{8}\right)$. Esto podría deberse a que la tonometría fue determinada en las mañanas, horas en que la PIO puede alcanzar sus valores más altos $(1.9 .10 .14)$.

Sólo un $6 \%$ de la población normal en los EE.UU. tiene una RCD mayor o igual a $0,5\left({ }^{14}\right)$. En comparacion a nuestro resultado. consideran que la evaluación del aspecto del nervio óptico es un parámetro difícil de evaluar incluso entre observadores exper1os $\left({ }^{15.16}\right)$, además que el registro de la RCD difiere si el método empleado ha sido la oftalmoscopía directa o fotografías esteroscópicas ('). Se ha postulado que el rastreo público de glaucoma basado exclusivamente en el aspecto del nervio optico resultaría más práctico y eficaz que la tonometría $\left({ }^{1.4}\right)$, incluso se sugiere un nuevo método en la clasificación del glaucoma basado en la apariencia del disco optico $\left({ }^{17}\right)$. El hallazgo de hemorragia de la papila ha sido muy frecuente en este estudio.

La tonometría ocular tuvo una sensibilidad regular del $75 \%$, to que concuerda con Shields $\left({ }^{1}\right)$, quien reporta entre 50 y $70 \%$; encontramos una especificidad de $84,47 \%$. Estos dos indicadores revclan que si determinamos la presion intrancular solamente, no se diagnosticaráan un $25 \%$ de casos de glaucoma.

Además, como existen casos falsos positivos, un porcentaje de la población que no tiene la enfermedad deberá someterse erróneamente a un costoso tratamiento. Asimismo, la evaluación del aspecto del nervio optico tiene una sensibilidau del $75 \%$ y especificidad del $93 \%$. Si enipleamos ambos parámetros mejoraremos la sensibilidad y especificidad de los programas de rastreo de glaucoma.

El campo visual es otro criterio importante que complementa la investigación, pero requiere una infratstructura de alto costo, una gran inversión de tiempo y un al to grado de colaboración por parte del paciente.

\section{CONCLUSIONES}

1.- La detección de glaucoma en campañas públicas de salud ocular basadas exclusivamente en la búsqueda de valores altos de presión intraocular no es confiable. Si evaluamos además el aspecto del nervio óptico. que es un procedimiento rápido y de bajo costo, se mejora la sensibilidad y especificidad de los programas de rastreo de glaucoma.

2.- Alrededor del $25 \%$ de pacientes con glaucoma de presión normal no serian detectados en campañas de detección que emplean exclusivamente la tonometría ocular.

3.- La distribución de la presión intraocular y la relación copa/disco entre ambos ojos y sexos es sinilar.

4.- A mayor edad existe un incremento significativo de la relación copa/disco.

5.- A mayor presión intraocular existe un incremento signilicativo de la relación copaddisco.

6.- La frecuencia de pacientes con hipertensión intraocular es mayor que la reportada en la literatura.

7.- La casuística de glaucoma crónico $(1,96 \%)$ y de pacientes con excavación pseudoglaucomatosa del disco $(6,71 \%)$ es comparable a la de otras investigaciones.

\section{BIBLIOGRA FÍA}

1) Shields MB. Texbook of glaucoma. $2^{\text {dd }} \mathrm{ed}$. Baltimore: Williams \& Wilkins; 1987.

2) Ardiles C, Figuerola F, Lopez I, Wong C. Ceguera por Glaucoma. Rev Per Oft 1977: 7(1): 145-9.

3) Leske MC. The epimemiology of Open-angle glaucoma: A revieu. Am J Epidemiol 1983: 118: 166-91.

4) American Academy of Opthalmology. Primary Open-angle Glaucoma. Preferred Practice Paicru. San Francisco: American Academy of Ophtalmology: 1992.

5) Zarate A, Galvis V. Fundación oftalmológica de Santander. Ceguera en América Latina y en el mundo: Aproximación al problema. Seminario en Salud Ocular Comunitaria. Bucaramanga, Colombia: Christotfel Blindenmission: abril de 1994.

6) Wong C, Tenorio A, Ardiles C y col. Prevención de Cegueras por Glaucoma: Resultados y aspecios operativos de 14 campañas. Arch Per Oftalmol 1986: 1(1): 17-21.

7) Oyola I. Duspistaje de (iluucoma. Tesis de Doctor en Medicina. LPCH. Lima-Perú. 1976.

11) Rosas P, Villavicencio D. Hipertensión Ocular y glaucoma en Arequipa. Rev Per Ottalmol 1974: 6(2): 15-23.

8) Vargas E. Anatomia del Glaucoma: Cambios simélricos y asimétricos. Acta slel V Ciongreso Peruana de Oftalmología 12-16 nov. 1974. Rev Per Orialmol 1974: 6 (2): 13-4. 
9) Kitazawa Y, Horie T. Diurnal variation of intrancular pressure in primary open-angle glaucoma. Am I Ophtbal 1975: 79: 557 .

10) Ruiz .J, Wong C. El significado clínico de las variaciones diurnas de la lonometría ocular. Arch Per Ojtalmol 1994 Set; 6(1): 27-9.

13) Klein B, Klein R. Prevalence of glaucoma: The beaver Dam Eye study. Ophthalmolog: 1992: 99(10): 1499-1504.

12) Beaujon M, Bcaujon O. Exludio comparativo de la neumotonometría y tonometría por aplanación. Rev Oisalmol Vene\% 1979: 37(1): 94-108
14) American Academy of Ophthalmology. Basic and clinical science course, section 10: Glaucoma, lens and anterior segment trauma. San Francisco: AAO: 1992-93.

15) Trobe .J1, Glaser JS, Cassedy J, Herschler I, Anaderson R. Nonglaucomatous excavation of the optic disc. Arch ophthalmol 1980: $98: 1046-50$

16) Jonas JB, Zach FM, Gusck GC, Naumann GO. Psedoglaucomatous physiologic large cups. Am J Ophthalmol 1989: 107: 137-44.

17) Spacth G. A new classification of glaucoma including focal glaucoma. Surv Ophibalmol 1994 May: 38(Suppl): S9-S17.

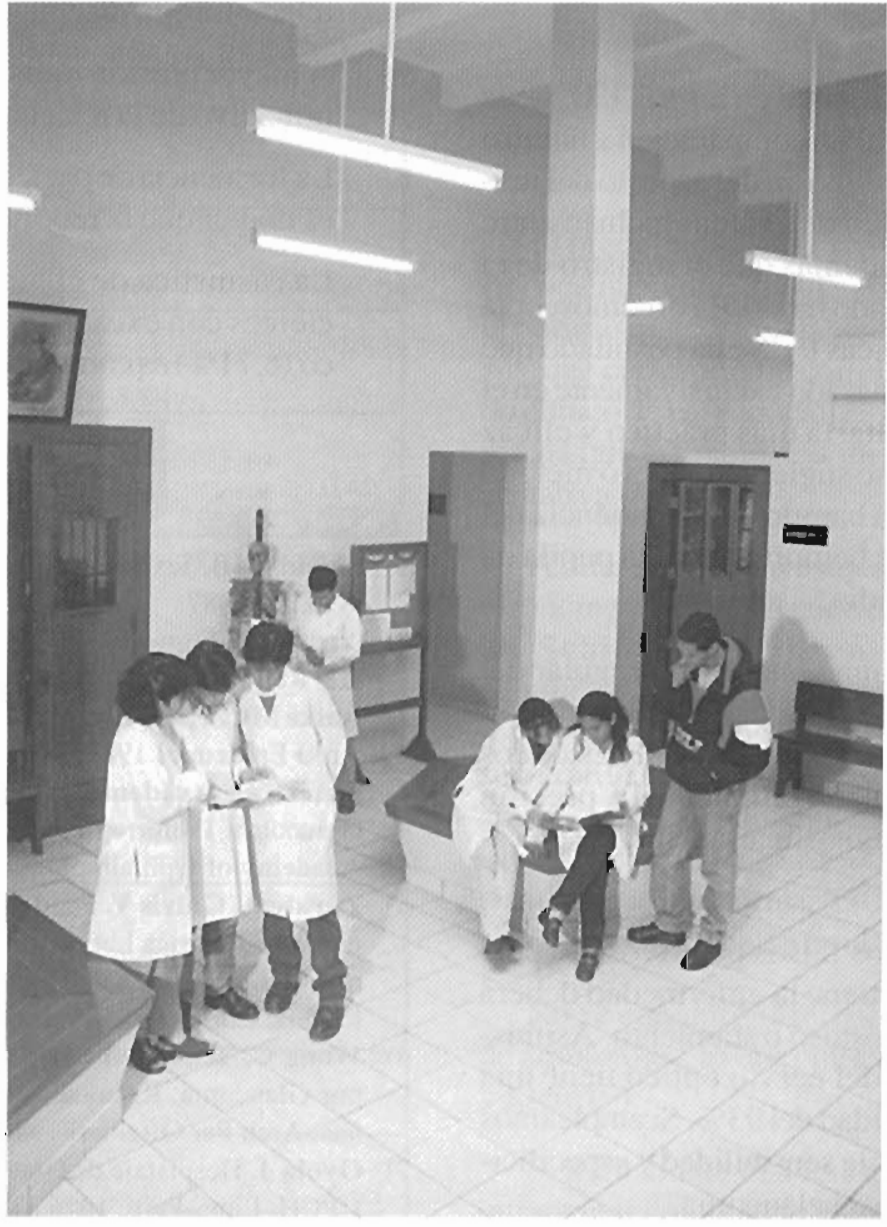

Patio del muevo pabellón de Fisiología. Faculad de Medicina de la UNMSM. Lima-Perí. (Aichivo Fotografico Univessiturio-TELEDUSM) 